\title{
Using Hilbert-Huang Transform to Assess EEG Slow Wave Activity During Anesthesia in Post-Cardiac Arrest Patients
}

\author{
Jukka Kortelainen, Member, IEEE, Eero Väyrynen, Usko Huuskonen, Jouko Laurila, Juha \\ Koskenkari, Janne T. Backman, Seppo Alahuhta, Tapio Seppänen, and Tero Ala-Kokko
}

\begin{abstract}
Hypoxic ischemic encephalopathy (HIE) is a severe consequence of cardiac arrest (CA) representing a substantial diagnostic challenge. We have recently designed a novel method for the assessment of HIE after CA. The method is based on estimating the severity of the brain injury by analyzing changes in the electroencephalogram (EEG) slow wave activity while the patient is exposed to an anesthetic drug propofol in a controlled manner. In this paper, Hilbert-Huang Transform (HHT) was used to analyze EEG slow wave activity during anesthesia in ten post-CA patients. The recordings were made in the intensive care unit 36-48 hours after the $\mathrm{CA}$ in an experiment, during which the propofol infusion rate was incrementally decreased to determine the drug-induced changes in the EEG at different anesthetic levels. HHT was shown to successfully capture the changes in the slow wave activity to the behavior of intrinsic mode functions (IMFs). While, in patients with good neurological outcome defined after a six-month control period, propofol induced a significant increase in the amplitude of IMFs representing the slow wave activity, the patients with poor neurological outcome were unable to produce such a response. Consequently, the proposed method offer substantial prognostic potential by providing a novel approach for early estimation of HIE after CA.
\end{abstract}

\section{INTRODUCTION}

C ARDIAC arrest (CA) is a condition in which the circulation of blood is ceased due to the failure of the

This work was financially supported by Medical Research Center Oulu (MRC Oulu), grant 40273/14 from Tekes - Finnish Funding Agency for Innovation, Orion Research Foundation, Instrumentarium science foundation, Emil Aaltonen foundation, Oulu University Scholarship Foundation, Finnish Foundation for Cardiovascular Research, and Finnish Science Foundation for Economics and Technology.

J. Kortelainen is with the Physiological Signal Analysis Team, Center for Machine Vision and Signal Analysis, University of Oulu, Finland and Department of Clinical Neurophysiology, Medical Research Center Oulu, University of Oulu and Oulu University Hospital, Finland (e-mail: jukortel@ee.oulu.fi).

E. Väyrynen and T. Seppänen are with the Physiological Signal Analysis Team, Center for Machine Vision and Signal Analysis, University of Oulu, Finland.

U. Huuskonen is with the Department of Clinical Neurophysiology, Medical Research Center Oulu, University of Oulu and Oulu University Hospital, Finland.

J. Laurila, J. Koskenkari and T. Ala-Kokko are with the Unit of Surgery, Anaesthesia and Intensive Care, Medical Faculty, University of Oulu, Finland and Division of Intensive Care Medicine, Medical Research Center Oulu, University of Oulu and Oulu University Hospital, Finland.

J. T. Backman is with the Department of Clinical Pharmacology, University of Helsinki and Helsinki University Hospital, Finland.

S. Alahuhta is with the Unit of Surgery, Anaesthesia and Intensive Care, Medical Faculty, University of Oulu, Finland and Department of Anaesthesiology, Medical Research Center Oulu, University of Oulu and Oulu University Hospital, Finland. heart to contract effectively. Approximately 360,000 out-ofhospital CAs occur in the USA alone each year [1]. While half of the patients admitted to hospital and intensive care unit (ICU) survive [2], a major part of the survivors suffer from permanent brain damage called hypoxic ischemic encephalopathy (HIE) due to global cerebral ischemia during the CA [3]. Consequently, neurological complications represent a substantial source of costs and loss of quality of life being the leading cause of disability after CA. Incorporating the brain in any cardiopulmonary resuscitation and follow-up treatment strategies has thus been demanded in the recent guidelines of American Heart Association [4].

At the moment, there is no reliable technological solution for early estimation of HIE after CA. For example, regular imaging modalities such as computed tomography or magnetic resonance imaging do not reliably estimate the diffuse brain injury resulting from hypoxia. Electrophysiological measurements as well as specific serum proteins in addition to clinical examination provide today's golden standard for the assessment of HIE, giving, however, only a rough estimate of the damage [5],[6]. The lack of reliable estimation of HIE complicates the clinical decision making as well as the development of new treatments of this condition.

We have recently designed a novel method for the assessment of HIE after CA. The method is based on estimating the severity of the injury by analyzing changes in the electroencephalogram (EEG) while the patient is exposed to an anesthetic drug propofol in a controlled manner. According to our recent findings [7], hypoxic brain injury fundamentally disturbs the neural system responsible for the generation of slow waves, the most important EEG signatures of non-rapid eye movement sleep [8] also seen during general anesthesia [9]. By measuring the EEG response to propofol, this disturbance can be revealed and used as a prognostic tool for irreversible brain injury.

In this paper, Hilbert-Huang Transform (HHT) [10], an adaptive data-driven method designed for the analysis of non-stationary signals, is used to assess EEG slow wave $(<1$ $\mathrm{Hz}$ ) activity during anesthesia in post-CA patients. We have previously shown the method to be able to extract stable signal components representing slow wave activity from EEGs of neurologically healthy patients during propofol anesthesia [11]. Here we investigate whether these signal components can be extracted from the data collected in the ICU during controlled propofol exposure and utilized in the 
estimation of the severity of HIE. The paper is organized so that Section II describes the data collection procedure and the EEG analysis. The results are given in Section III. In Section IV, the conclusions of the study are presented.

\section{MATERIALS AND METHODS}

\section{A. Experimental Protocol}

The study was approved by The Regional Ethics Committee of the Northern Ostrobothnia Hospital District. Ten comatose patients resuscitated from out-of-hospital CA participated in the study. Patients with earlier disease affecting the central nervous system were excluded. The patients had received hypothermia treatment as a neuroprotective measure before the experiment, which was carried out $36-48 \mathrm{~h}$ after the CA while the patients were still sedated with propofol.

In the experiment, EEG was recorded with 19-channel electrode cap according to the 10/20 international system using Nicolet nEEG Modular Neurodiagnostic System with a v32 Amplifier. A sampling frequency of $500 \mathrm{~Hz}$ and bandwidth of $0.053-125 \mathrm{~Hz}$ was used and the signals were referenced to common average. During the experiment, the propofol infusion rate was incrementally decreased
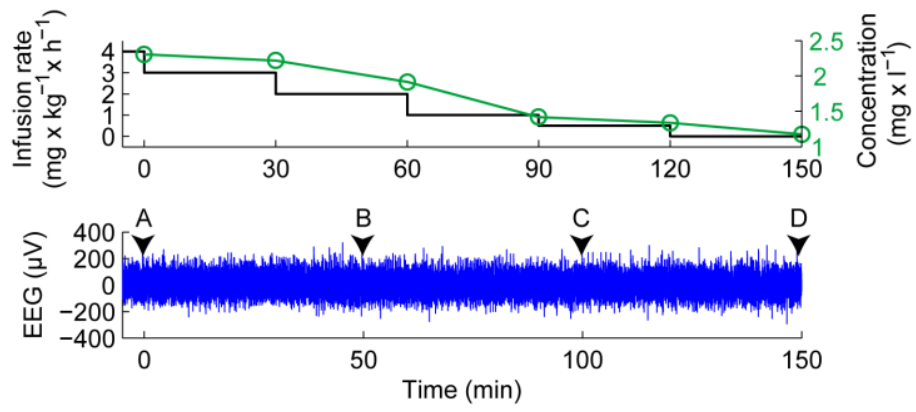

A

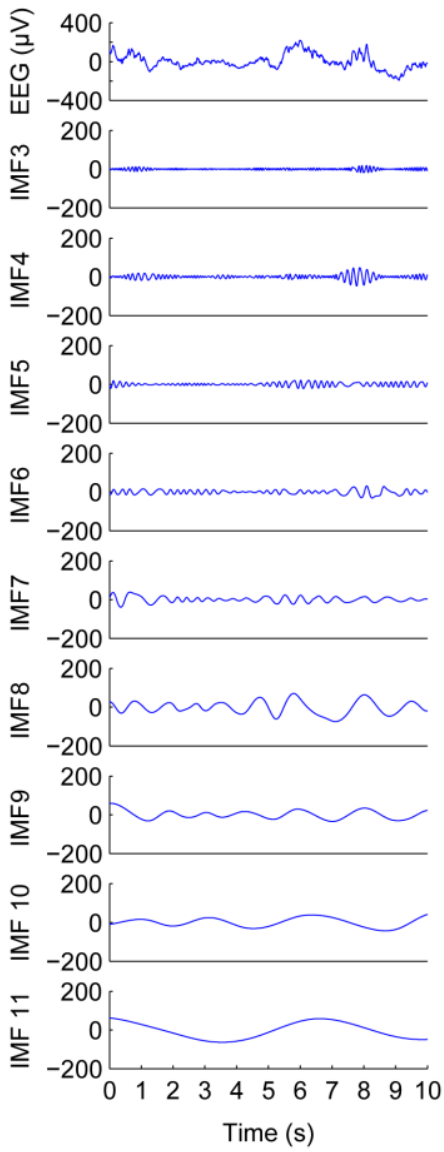

B

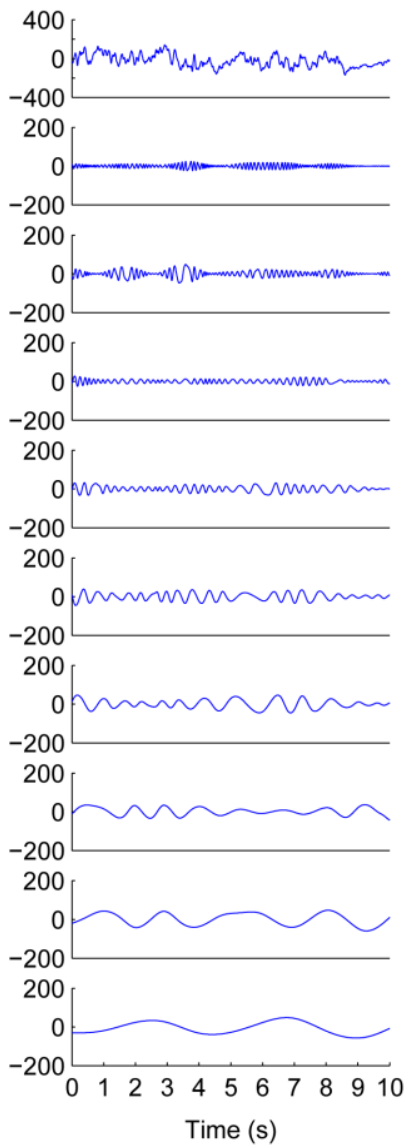

C

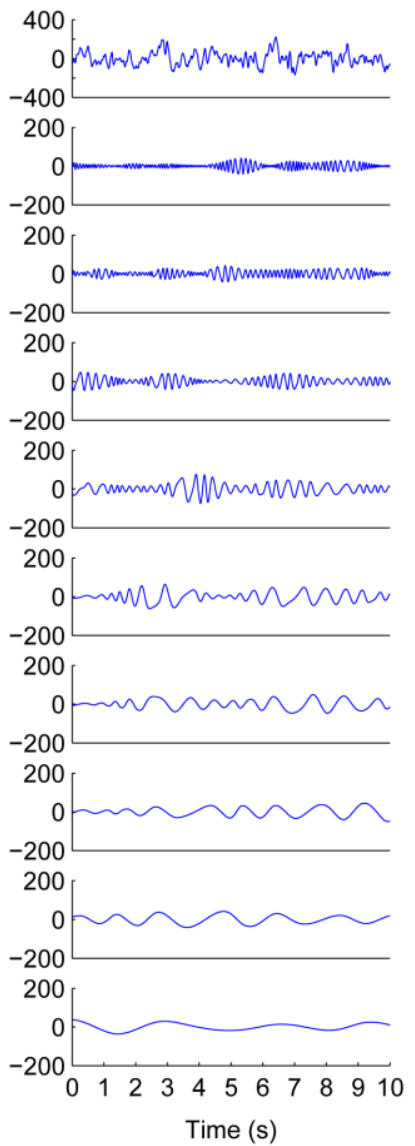

D

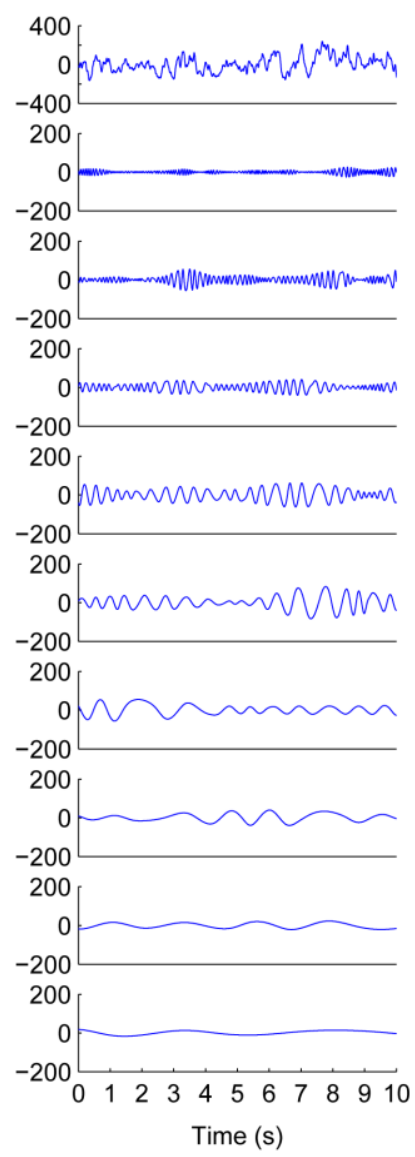

Fig. 1. The propofol infusion rate, blood concentration and EEG during the experimental protocol. Above, the propofol infusion rate (black line) and blood concentration (green line) is given in relation to the EEG (blue data) during the entire experimental protocol for one patient with good outcome. The infusion rate was decreased step-wise every $30 \mathrm{~min}$ and the propofol concentration was determined on every step just before the next decrease as well as in the end of the experiment (green circles). Below, short EEG signal samples and the corresponding IMFs (3-11) at four different phases (A-D) of the experiment are presented. 
following a predefined protocol (Fig. 1) to determine the drug-induced changes in the EEG at different anesthetic levels. The decrease was started from the highest acceptable infusion rate during the intensive care $\left(4 \mathrm{mg} \times \mathrm{kg}^{-1} \times \mathrm{h}^{-1}\right)$ and continued step-wise every $30 \mathrm{~min}$ until the drug administration was finally switched off. Same infusion rates $\left(4,3,2,1,0.5\right.$, and $\left.0 \mathrm{mg} \times \mathrm{kg}^{-1} \times \mathrm{h}^{-1}\right)$ were used for all patients. To determine the propofol concentration, a blood sample was taken before each change in the infusion rate as well as in the end of the experiment, i.e. 30 min after turning propofol off (Fig. 1). The severity of the HIE hypoxicischemic brain injury was determined by evaluating the neurological recovery of the patient six months after the CA using the Cerebral Performance Category (CPC). Based on this, the patients were assigned to either good (CPC 1-2) or poor (CPC 3-5) outcome group.

\section{B. EEG Analysis}

The signal processing was carried out with Matlab technical computing language, version 2011b (The MathWorks, Inc., Natick, MA). In the study, only the data from channel Fp1 were used. This frontal channel was selected as our earlier study showed pronounced EEG responses to the anesthetic in the prefrontal area [7]. For the EEG analysis, 5-min signal samples were picked just before each decrease of the drug infusion rate and in the end of the experiment corresponding to the collection of the drug concentration blood samples. From each 5-min signal sample a 30 -s representative artifact-free sequence was selected for further analysis. The signals were then filtered using a low-pass FIR filter with a cutoff frequency of $48 \mathrm{~Hz}$ to reduce high-frequency noise.
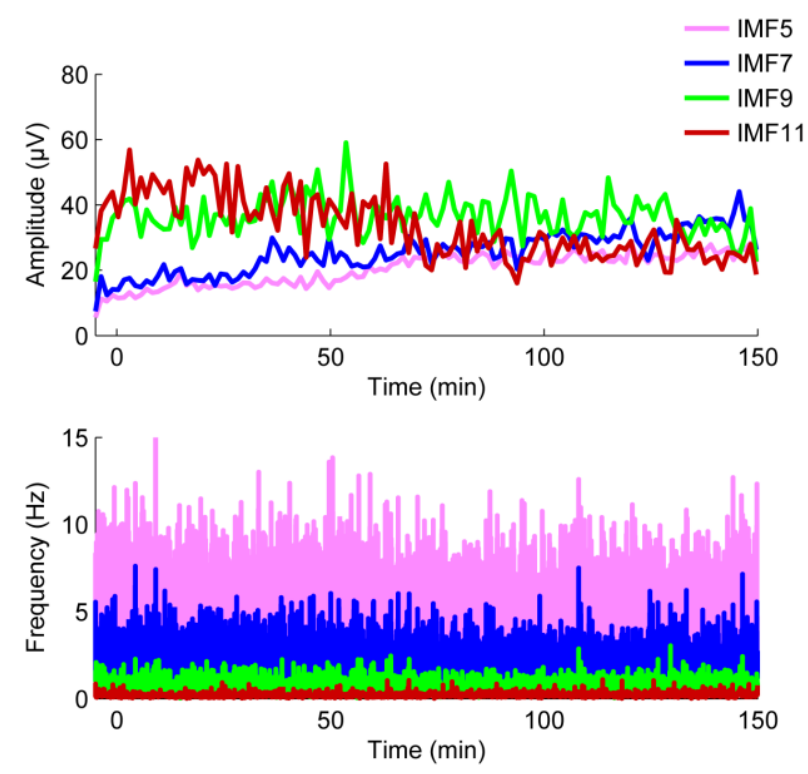

Fig. 2. Instantaneous amplitude (higher row) and frequency (lower row) of intrinsic mode functions (IMFs) 5, 7, 9, and 11 for one patient with good outcome. The IMFs are extracted from the EEG recorded during the entire experiment. As in Fig. 1, time point zero represents the first decrease in the propofol infusion rate.
To investigate the dynamical changes in the signal characteristics, HHT was applied to the EEG data. First, the method was applied to the entire EEG recording of one patient to illustrate the signal changes as a function of time. Then, the method was applied to the 30-s signal sequences representing different propofol infusion rates to examine the differences between the good and poor outcome groups in the whole dataset. The HHT algorithm contains two principal parts:

1. Empirical Mode Decomposition (EMD)

2. Hilbert Spectral Analysis (HSA)

In EMD, the signal is iteratively decomposed into components called intrinsic mode functions (IMFs) each component containing lower frequency oscillations than the preceding one. In HSA, Hilbert transform is used to compute the instantaneous frequency and amplitude of different IMFs. A more detailed description of the HHT applied is given in [11].

\section{RESULTS}

In Fig. 1, the propofol infusion rates, blood concentrations and EEG during the entire experimental protocol are presented for one patient with good outcome. In addition, short EEG signal samples and the corresponding IMFs at four different phases of the experiment are presented. Lowering the infusion rate decreased the amount of drug in the blood which consequently is seen in the EEG as a reduced anesthetic effect. In signal characteristics, this means a shift of power from lower frequencies (IMFs 8-11) to higher frequencies (IMFs 3-7). When applied to this entire EEG recording, EMD extracted a total of 23 IMFs. Fig. 2 presents the instantaneous frequencies and amplitudes of IMFs $5,7,9$, and 11 calculated for the same recording. The shift of activity from lower frequencies to higher frequencies

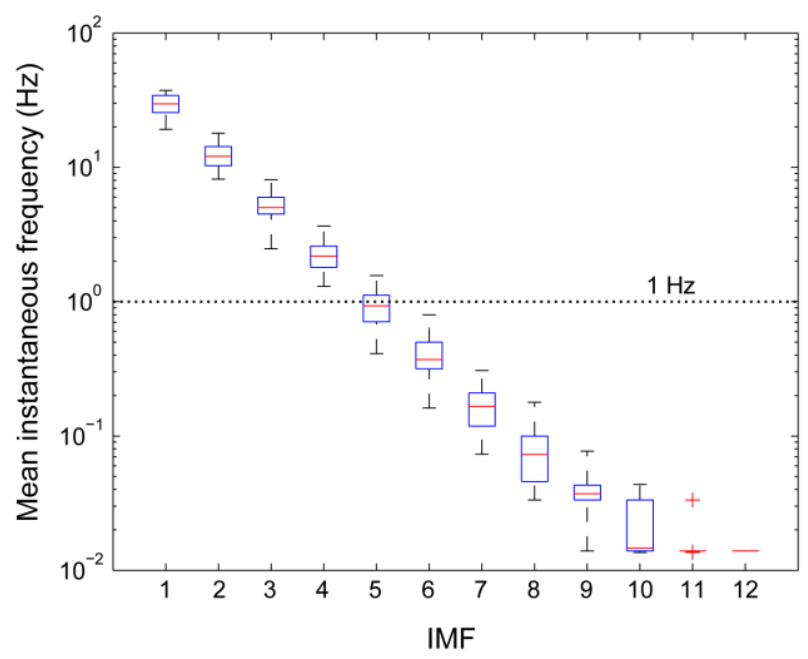

Fig. 3. The mean instantaneous frequencies of different intrinsic mode functions (IMFs) calculated for the 30 -s signal samples. The values are given in logarithmic scale and each box shows the median, range, and $25^{\text {th }}$ and $75^{\text {th }}$ percentiles. 


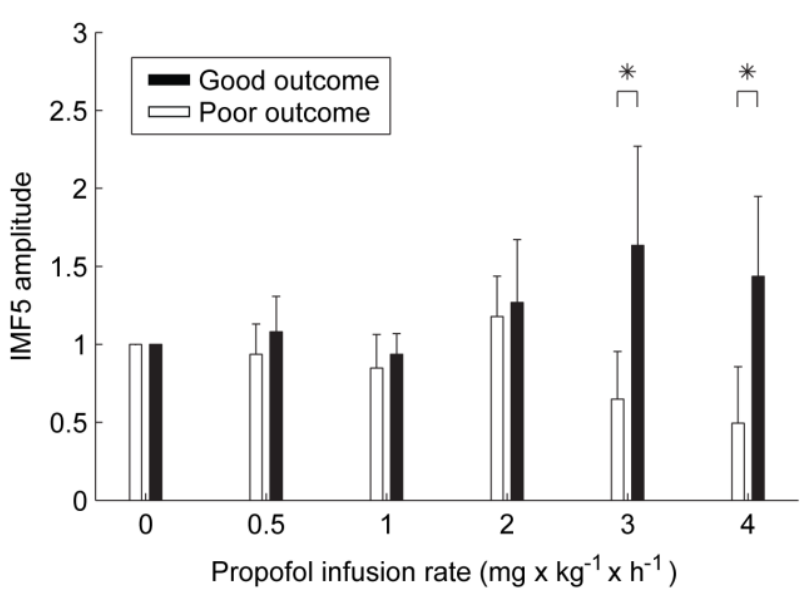

Fig. 4. The effect of propofol on the amplitude of intrinsic mode function (IMF) 5 in patients with good and poor neurological outcome. The amplitudes are given at different propofol infusion rates for patients with good $(\mathrm{N}=6)$ and poor $(\mathrm{N}=4)$ neurological outcome. The values represent mean instantaneous amplitudes calculated for the 30 -s signal samples given relative to the individual value at propofol infusion rate $0 \mathrm{mg} \times \mathrm{kg}^{-1} \times \mathrm{h}^{-1}$. Data are means; error bars show SD. Asterisks indicate statistically significant difference between groups $\left({ }^{*} p<0.05\right.$, unpaired two-sample t-test).

is clearly seen in the behavior of IMFs of which 5 and 7 substantially increase their amplitude whereas the amplitude of IMF 11, representing slow wave activity, is halved.

The mean instantaneous frequencies of different IMFs calculated for the 30 -s signal samples at different anesthetic levels are given in Fig. 3. For these shorter signal sequences, 9-12 IMFs were extracted by EMD depending on the sample. As shown in the figure, the slow wave activity was captured mostly by IMF 5. Fig. 4 presents the effect of propofol on the amplitude of IMF 5 in patients with good and poor neurological outcome. In the patients with good neurological outcome $(\mathrm{N}=6)$, the amplitude of IMF 5 representing the slow wave activity was found to be strongly affected by propofol being substantially larger at high infusion rates. On the contrary, the patients with poor neurological outcome $(\mathrm{N}=4)$ were unable to generate propofol-induced slow waves measured by the amplitude of IMF 5. Consequently, propofol-induced change in the amplitude of IMF 5 was statistically significantly different between the two groups at high infusion rates ( 3 and $4 \mathrm{mg} \times$ $\left.\mathrm{kg}^{-1} \times \mathrm{h}^{-1}\right)$.

\section{CONCLUSIONS}

As a conclusion, HHT was used to analyze EEG slow wave activity during anesthesia in ten post-CA patients. The recordings were made in the intensive care unit 36-48 hours after the CA in an experiment, during which the propofol infusion rate was incrementally decreased to determine the drug-induced changes in the EEG at different anesthetic levels. HHT was shown to successfully capture the changes in the slow wave activity to the behavior of IMFs. While, in patients with good neurological outcome defined after a six- month control period, propofol induced a significant increase in the amplitude of IMFs representing the slow wave activity, the patients with poor neurological outcome were unable to produce such a response. Consequently, the proposed method offer substantial prognostic potential by providing a novel approach for early estimation of HIE after CA.

\section{REFERENCES}

[1] A. S. Go, D. Mozaffarian, V. L. Roger et al., "Executive summary: heart disease and stroke statistics--2014 update: a report from the American Heart Association," Circulation, vol. 129, pp. 399-410, 2014.

[2] M. S. Eisenberg and T. J. Mengert, "Cardiac resuscitation," N. Engl. J. Med., vol. 344, pp. 1304-1313, 2001.

[3] G. B. Young, "Clinical practice. Neurologic prognosis after cardiac arrest," N. Engl. J. Med., vol. 361, pp. 605-611, 2009.

[4] C. W. Callaway, M. W. Donnino, E. L. Fink et al., "Part 8: PostCardiac Arrest Care: 2015 American Heart Association Guidelines Update for Cardiopulmonary Resuscitation and Emergency Cardiovascular Care," Circulation, vol. 132, pp. S465-82, 2015.

[5] E. G. Zandbergen, R. J. de Haan, C. P. Stoutenbeek, J. H. Koelman, and A. Hijdra, "Systematic review of early prediction of poor outcome in anoxic-ischaemic coma," Lancet, vol. 352, pp. 1808-1812, 1998.

[6] M. Tiainen, R. O. Roine, V. Pettila, and O. Takkunen, "Serum neuronspecific enolase and S-100B protein in cardiac arrest patients treated with hypothermia," Stroke, vol. 34, pp. 2881-2886, 2003.

[7] J. Kortelainen, E. Väyrynen, U. Huuskonen et al., "Propofol-induced slow waves - A pharmacological test for brain dysfunction," Anesthesiology, submitted for publication.

[8] V. Crunelli, F. David, M. L. Lorincz, and S. W. Hughes, "The thalamocortical network as a single slow wave-generating unit," Curr. Opin. Neurobiol., vol. 31C, pp. 72-80, 2014.

[9] P. L. Purdon, E. T. Pierce, E. A. Mukamel et al., "Electroencephalogram signatures of loss and recovery of consciousness from propofol," Proc. Natl. Acad. Sci. U. S. A., vol. 110, pp. E1142-51, 2013.

[10] Huang NE, Shen Z, Long S et al., "The empirical mode decomposition and the Hilbert spectrum for nonlinear and non-stationary time series analysis," Proc. R. Soc., vol. 454, 1998.

[11] J. Kortelainen and E. Vayrynen, "Assessing EEG slow wave activity during anesthesia using Hilbert-Huang Transform," Conf. Proc. IEEE Eng. Med. Biol. Soc., vol. 2015, pp. 117-120, 2015. 